\title{
Photonic crystals as host material for a new generation of microwave components
}

\author{
F. Daschner ${ }^{1}$, R. Knöchel ${ }^{1}$, E. Foca ${ }^{2}$, J. Carstensen ${ }^{2}$, V. V. Sergentu ${ }^{3}$, H. Föll ${ }^{2}$, and I. M. Tiginyanu ${ }^{3}$ \\ ${ }^{1}$ Microwave Laboratory, Christian-Albrechts-University of Kiel, Kaiserstr. 2, 24143 Kiel, Germany \\ ${ }^{2}$ Chair for General Materials Science, Christian-Albrechts-University of Kiel, Kaiserstr. 2, 24143 Kiel, Germany \\ ${ }^{3}$ Institute of Applied Physics, Academy of Sciences of Moldova, 5 Academy str., 2028 Chisinau, Moldova
}

\begin{abstract}
In order to verify simulations of a concave lens based upon a photonic crystal, it was scaled and built for the application in the microwave range. Its field distribution was measured between 5.5 and $12 \mathrm{GHz}$. Due to the effective refractive index smaller than 1, focusing points were found in spite of the concave shape. The survey of the field distributions at the investigated frequencies leads to other possible applications of photonic crystals in the microwave and millimetre wave region, like beam-splitter and prism.
\end{abstract}

\section{Introduction}

Photonic Crystals (PC) are artificial materials that give the possibility to control the propagation of electromagnetic waves (Joannopoulos et al., 1999). Generally they will form periodic dielectric structures with a certain lattice constant $a$ which is one of the parameters allowing the control of propagation properties of the PC. One can design a PC in such a way that for particular frequencies no propagation is possible or the propagation paths are modified strongly. The main focus of this paper, however, is the description of experiments with some primary considerations for the possible applications of the presented structures.

In the present work we treat new microwave components based on those PC that have an effective index of refraction $n_{\text {eff }}<1$. That is, under certain circumstances, PC can be viewed as homogenous materials described by an effective material parameter, although being highly anisotropic and dispersive. However, this is only possible for the case that the dielectric contrast in the dielectric constant is high enough (Notomi, 2000). More precisely we focus our attention on possible lenses based on such PC. In the virtue of their refractive index these lenses would have a concave shape in order to be able to focus the radiation.

Correspondence to: F. Daschner

(fd@tf.uni-kiel.de)

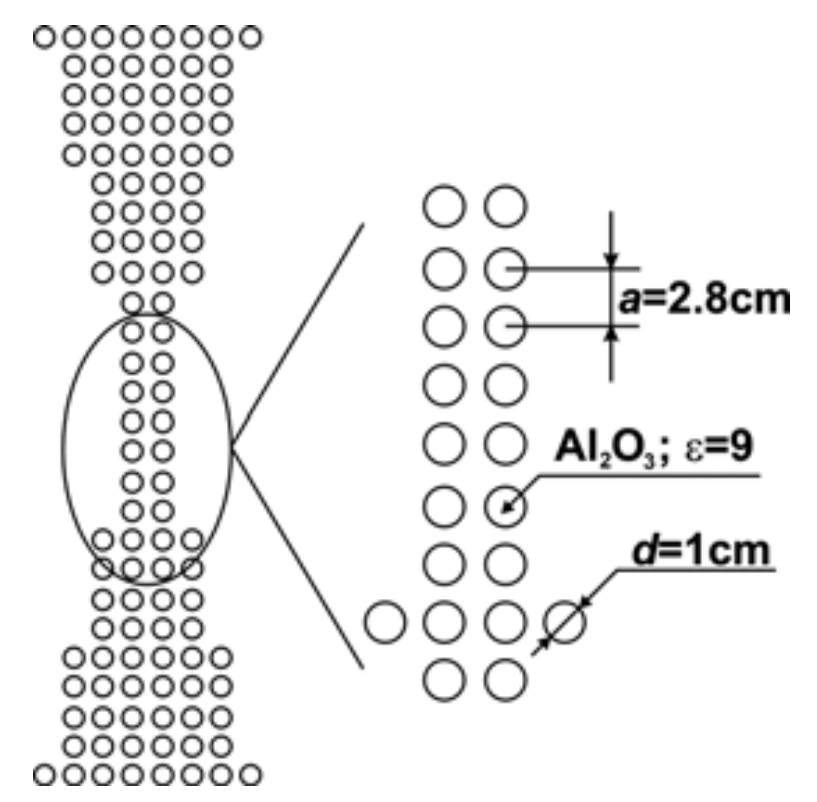

Fig. 1. Top view of the manufactured concave lens; structural dimensions are indicated.

\section{Experiments}

The PC used as "bulk" for the lens presented here consisted of an periodic arrangement of alumina cylinders, i.e. formed a 2-D structure. For the frequency space involved in the presented measurements alumina has a dielectric constant $\varepsilon=9$. The lattice constant of the PC is $a=2.8 \mathrm{~cm}$ and the radius of the rods is $r=0.5 \mathrm{~cm}$. Generally the theoretical calculations of the properties of a PC are completely correct only for a PC having infinite dimensions. In practical applications a limitation of the size is needed. For the experiments described here a lens was built using only 112 dielectric rods, arranged in a square lattice as shown in Fig. 1. Due to the small size, the set-up is not any longer called "photonic crystal" in this text,

Published by Copernicus GmbH on behalf of the URSI Landesausschuss in der Bundesrepublik Deutschland e.V. 


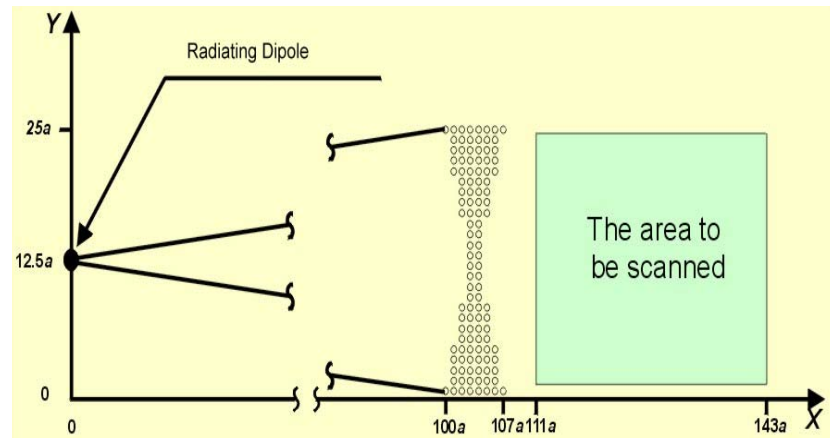

Fig. 2. Drawing of the Measurement setup. The field distribution was measured in a constant-z-plane.

but "lens". It has a concave shape and for frequencies where $n_{\text {eff }}<1$ a focusing point should be observed.

In order to approximate an incident homogenous, plane wave at the frontal surface of the lens, a dipole was placed at a distance of $100 a=2.8 \mathrm{~m}$. in front of it. The sending dipole was connected to an HP83752B synthesized sweeper with a power of $16 \mathrm{dBm}$. In order to measure the electro-magnetic field distribution behind the lens, a receiving antenna was mounted on a xyz-table (see Figs. 2 and 3). It was moved over an area of $700 \mathrm{~mm}$ by $800 \mathrm{~mm}$ and every $5 \mathrm{~mm}$ the field was measured. Hence, the total number of points measured was 22701 .

The received signal was amplified by an HP83051A microwave system amplifier. This amplified signal was fed into an ACSP2506-SN0898 detector diode. Since the source signal was amplitude modulated, the detected signal had a frequency of $1 \mathrm{kHz}$. This signal was processed by a Stanford Research Systems SR530 lock-in amplifier. The time constant used for the filter was selected to $300 \mathrm{~ms}$, hence the sensitivity of the field scanner was relatively high. The whole system was controlled automatically by a microcomputer.

Because the measurement of the field distributions may be distorted by external signals and interferences caused by reflections, the setp-up was placed into an anechoic chamber. The field distributions were measured from $\lambda=2.5$ to $5.5 \mathrm{~cm}(12 \mathrm{GHz}$ down to $5.45 \mathrm{GHz})$. The polarizations measured were TE (the magnetic field components of the incident wave are in parallel to the dielectric rods) and TM (electric field parallel to rods). Doing this, the direction of the sending and receiving dipole was turned and the measurement procedure was done again.

In spite of the high effort using an anechoic chamber, one cannot completely avoid reflections: the distortion of the electromagnetic field distribution has to be considered. Moreover the dipoles ( $35 \mathrm{~mm}$ length) exhibit a certain frequency characteristic. Therefore it was decided to relate the measurements $(E(x, y))$ to free space measurements without the lens $\left(E_{0}(x, y)\right)$. The performance of the lens is expressed using a ration called intensity gain defined as

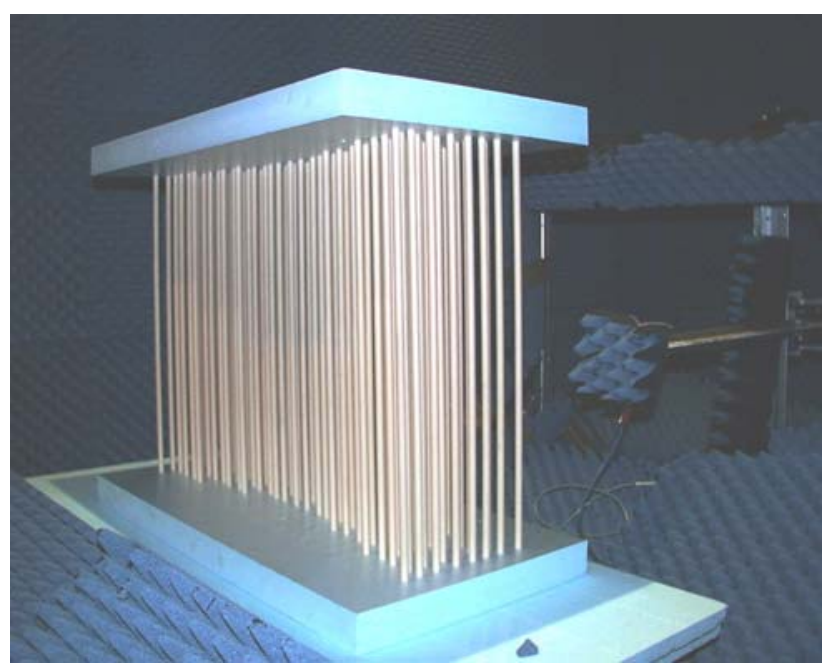

Fig. 3. Photograph of the investigated lens. The lens was placed in an anechoic chamber in front of a field scanner.

$I G=E_{(x, y)}^{2} / E_{0(x, y)}^{2}$.

\section{Results}

Figure 4 shows some selected field distributions. Thereby the simulations are confronted with the measurements. A good qualitative and quantitative identicalness can be seen. This implicates firstly that the simulations descried in Foca et al. (2005a,b) are successfully verified with real measurements. This agreement between simulations and measurements can be observed for all measured wavelengths. In case (a) $(a / \lambda=0.96, \mathrm{TM})$ and at many other wavelength a fuzzy field distribution can be observed. In case (b) $(a / \lambda=0.78$, TE) the lens works as a beam-splitter. A lens should have a focusing point and it can bee found in (c) $(a / \lambda=0.78$, TM). More features of the same structure could be found elsewhere (Foca et al., 2005a,b).

The frequency characteristics of the intensity gain of both investigated polarizations are shown in Fig. 5. The highest values found for the IG are 5.5 (TE) and 6.5 (TM). This is not even high, but one has to bear in mind that the lens is only one dimensional. For the two dimensional case (the lens would be built here using a 3 dimensional PC), this values has to be squared. Furthermore the lens has only the size of a few wavelengths (here 13..29, depending on the measured wavelength). Hence in comparison to optical lenses the area illuminated is very small.

The quality of focusing is not only addressed by the IG value, but also by the focus spot size. In order to assess this point, the measured intensity distribution in the focal spot was approximated by a bell shape function (i.e. a 2-D Gaussian function in this case). The focal spot area was defined as the full width at half maximum (FWHM). According to 


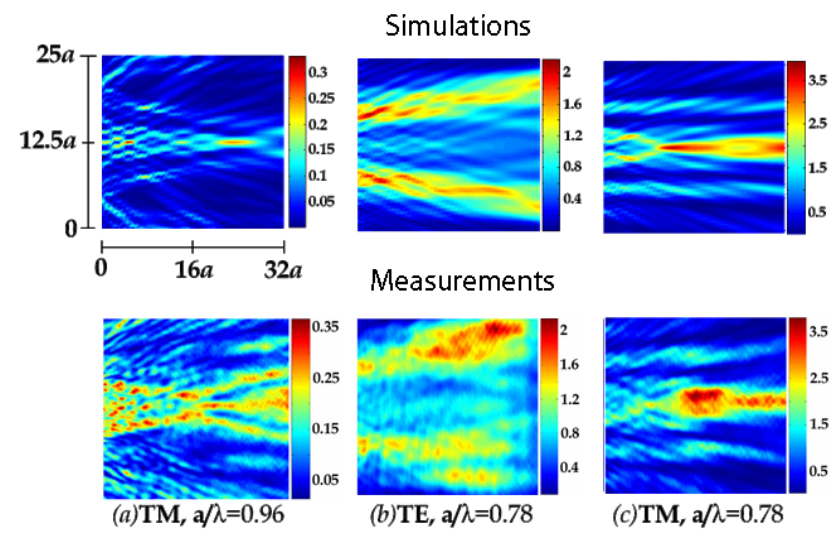

Fig. 4. (a) Simulations agree well with experiments; even for the case of a "fuzzy" distribution of the field; (b) For a certain wavelengths bands the beam will be split for the TE polarization; (c) Good focusing is found for the TM polarization.

Pendry (2000), "flat" lenses made form a negative index material could surpass this limit, but as the experiment seems to indicate, the concave lens investigated here can also overcome this limit at least for the TE polarization; while in the TM polarization it reaches this limit. The smallest ratio of the measured focusing area to the $\lambda^{2}$ is 0.24 for the TE polarization, occurring at $a / \lambda=0.58$, and 1.02 for the TM polarization, occurring at $a / \lambda=0.7$. The origin of the subwavelength focusing phenomenon is still questionable. We think that in our case this is rather related to the deterioration of the beam front at the exit of the lens and participation of the evanescent waves in the focus formation. This can be partially explained by the so called Zernike effect. However, due to the lack in space we do not go here in too many details since the generic Zernike effect will have its limitations in this case and more sophisticated discussions are needed in order to address all related issues. More results could be seen in Foca et al. (2006).

For the lack of space not all field distributions measured can be shown here. But there is another interesting behavior observed in the range between $\lambda=3.1 \mathrm{~cm}$ and $\lambda=3.6 \mathrm{~cm}$ (TM): the focusing point moves along the symmetry axis $(y=12.5 a)$. Therefore an application of the lens as a prism is thinkable. However, for this purpose the lens was not designed and an the arrangement may be still considerably improved if a prism design is intended.

\section{Conclusions}

The primary objective of the measurements described was to verify the simulations done with a concave lens made from dielectric rods. The measurements are in well agreement with the simulation and focusing points were found for the TE-polarization as well for the TM-polarization. Beside this, a qualitative view on the measured electromagnetic field dis-
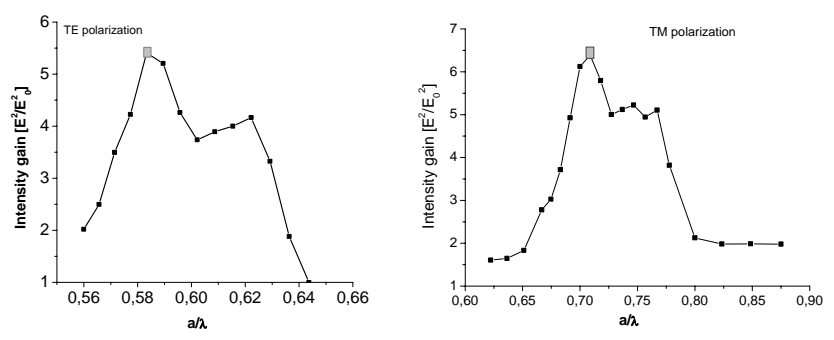

Fig. 5. Frequency Characteristic of the intensity gain of the TE- and TM-polarization.

tributions leads to other possible applications. For some frequencies, the lens works as a beam splitter. The band gap can be used for filter applications. Furthermore, in a specific range, the set-up works like a prism.

However, due to the huge degrees of freedom designing such arrangements (shape, lattice constant, dielectric contrast), the challenge is to find an optimal photonic crystal for a given task. For the use in optical electronic circuits, many applications are imaginable. Components based upon photonic crystals may help to close the terahertz-gap in the future, when nano-technologies will make it possible to manufacture such small PC. Because PC are scaleable, theoretically all frequency ranges are applicable.

In the microwave region their behavior can be studied in detail due to the manageable size. But many interesting components in the micro- and mm-wave range could also be developed using photonic crystals.

\section{References}

Joannopoulos, J. D., Meade, R. D., and Winn, J. N.: Photonic Crystals: Modeling the Flow of Light, Princenton Univ. Press, Princenton, NJ, 1995.

Notomi, M.: Theory of light propagation in strongly modulated photonic crystals: Refractionlike behavior in the vicinity of the photonic band gap, Phys. Rev. 62, 10696-10 705, 2000.

Felbacq, D., Tayeb, G., and Maystre, D.: Scattering by a random set of parallel cylinders, J. Opt. Soc. Am., 11, 2526, 1994.

Foca, E., Föll, H., Daschner, F., Sergentu, V. V., Carstensen, J., Knöchel, R., and Tiginyanu, I. M.: Efficient focusing with an Ultra-Low Effective-Index Lens based on Photonic Crystals, Proc. of the MRS Spring Meeting 2005, Symposium D, 2005a.

Foca, E., Föll, H., Daschner, F., Sergentu, V. V., Carstensen, J., Frey, S., Knöchel, R., and Tiginyanu, I. M.: Efficient focusing with a concave lens based on a photonic crystal with an unusual effective index of refraction, Phys. Stat. Sol., 202, 35, $2005 \mathrm{~b}$.

Foca, E., Föll, H., Carstensen, J., Sergentu, V. V., Tiginyanu, I. M., Knöchel, R., and Daschner, F.: Strongly frequency dependent focusing efficiency of a concave lens based on two-dimensional photonic crystals, Appl. Phys. Lett., 88, 011102, 2006.

Pendry, J. B.: Negative refraction makes a perfect lens, Phys. Rev. Lett., 85, 3966-3968, 2000. 